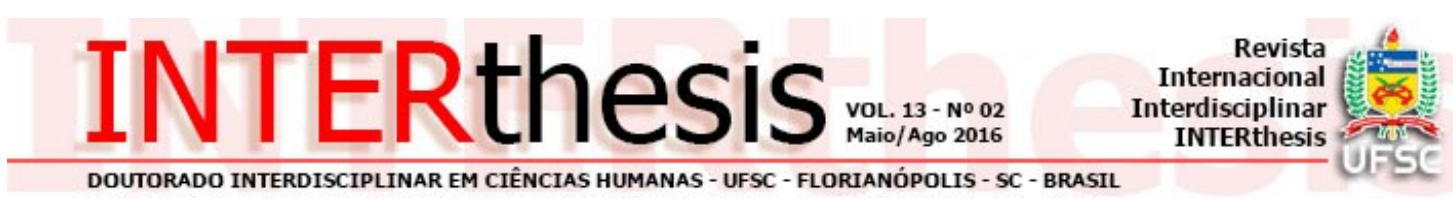

\title{
TEORIA DE SISTEMAS AUTOPOIÉTICOS Y LA "ACTOR NETWORK THEORY": ¿HACIA UNA CONVERGENCIA POSESTRUCTURALISTA?
}

Josep Pont Vidal ${ }^{1}$

\section{Resumen:}

Desde hace unos años se manifiesta un creciente interés entre los sociólogos en establecer un nexo entre la Teoría de sistemas sociales autorreferenciales de Niklas Luhmann con los marcos teóricos que componen la filosofía social posestructuralista. Aunque existe una autodefinición común estructural-funcionalista aparecen insoslayables diferencias entre la teoría sistémica de Luhmann y el constructivismo de la ANT.

Palabras clave: Teoría de sistemas autopoiéticos. Actor Network Theory. Comunicaciones. Actor.

\section{TEORÍA DE SISTEMAS AUTOPOIÉTICOS Y DIÁLOGOS CON EL POSESTRUCTURALISMO}

Con la popularización de conceptos nuevos en las ciencias sociales a mediados de la década de los años 1980, se dio paso a los que algunos denominaron New Systems Theory (BAILEY, 1994). Los debates sobre esta nueva orientación sistémica giraron en torno a observaciones de los "Real systems", direccionados a la observación de los sistemas naturales en los que se constataron variables dinámicas y estáticas y las posibilidades que ofrecen en términos de controlabilidad, accesibilidad y observabilidad y el estado estático. Los resultados no permanecieron desapercibidos para la teoría de sistemas y se dirigieron a analizar las posibilidades de implementación de la System and Control Theory en diversos campos, siendo sustentada en cálculos matemáticos y alogarítmicos.

La obra y la teoría de sistemas sociales o autorreferenciales de Niklas Luhmann se inserta en el contexto cambio paradigmático de esta década, y también

1 Doutor em Sociologia Política pela Universidad de Barcelona, Espanha. Professor adjunto e pesquisador do Núcleo de Altos Amazônicos e Coordenador do Programa de Pós-Graduação em Gestão Pública e Desenvolvimento na Universidade Federal do Pará, Belém, Pará, Brasil. E-mail: joseppontvidal@gmail.com

R. Inter. Interdisc. INTERthesis, Florianópolis, v.13, n.2, p.38-57 Mai-Ago. 2016 
en el cuestionamiento y paulatino distanciamiento de la teoría funcionalista, de los paradigmas de la unidad, y de las ambigüedades de la Teoría General de Sistemas en la definición de sistema, y se dirige hacia un pensamiento que supone un nuevo punto de partida y la renuncia de un centro o punto de partida. Luhmann realiza una transición de la Teoría de sistemas abiertos a los sistemas autorreferenciales, con lo que fue posible entender cómo un sistema podía mantener sus límites a pesar de su dependencia de reproducción comunicativa con el entorno, aunque la clarificación fue causa de nuevos problemas teóricos derivados de la autorreproducción de los sistemas, cuya explicación sobrepasa los objetivos de este ensayo.

Estudiosos de la teoría sociopoiética luhmanniana han procurado soluciones a estos problemas al iniciar un diálogo con los filósofos sociales posestructuralistas. Fruto de este diálogo desde la perspectiva sistémica y con el objetivo de buscar analogías con el "constructivismo estructuralista", se destaca el análisis de los conceptos centrales de la teoría de Pierre Bourdieu y las similitudes entre el concepto de "habitus" (entendido como una red de relaciones objetivas entre posiciones objetivamente definidas), los "campos de acción" y el "sistema" autopoiético. Tomando como referencia estos presupuestos, se han analizado las ideas centrales de sentido, autopoiesis y cierre operativo y las semánticas de otros conceptos de la teoría luhmanniana, que más controversias han causado entre los pensadores sistémicos "ortodoxos" y "heterodoxos".

En la perspectiva de análisis luhmanniana, este diálogo no ha sido una tarea libre de dificultades, al no ser posible introducir ningún otro concepto en su teoría "sin antes hacer referencia o haber utilizado a otros conceptos" (TRÖNDLE, 2012). Otra dificultad ha residido en la imposibilidad de realizar alguna observación sin una autorreferencia previa al no ser posible ninguna autorreferencia sin establecer previamente la abstracción de la diferenciación sistémica frontera / límite.

Entre las teorías posestructuralistas, se destaca el punto de vista constructivista de la "Actor Network-Theory" (ANT) representada y desarrollada por filósofos sociales como Bruno Latour, Michel Callon y John Law, y que comparten una serie de premisas comunes con la teoría sociopoiética, y se autodefinen como estructural-funcionalistas. Ambas perspectivas (la teoría sociopoiética luhmanniana y el punto de vista de la ANT), son consideradas "alternativas" al funcionalismo y al

${ }^{2}$ Bourdieu utilizó este criterio para definir su modelo sociológico.

R. Inter. Interdisc. INTERthesis, Florianópolis, v.13, n.2, p.39-57 Mai-Ago. 2016 
positivismo clásicos. Entre la comunidad de sociólogos se las ha acusado de deshumanizar a los humanos cuando igualan a los actores no-humanos a cosas. En el ámbito práctico y empírico de las aportaciones más sobresalientes de las teorías posestructuralistas, destacan en el área de las teorías organizacionales, resaltando dos cuestiones recurrentes y que han mostrado obstáculos teóricos y controversias en ambas perspectivas: ¿se manifiesta una convergencia semántica en el concepto de actor?, ¿cómo se puede transferir la idea de actor y sentido sin la predeterminación de seres humanos?, ¿es posible asumir la idea de autopoiesis en los nudos que forman una red?

En base a estas cuestiones, en este ensayo nos proponemos: en primer lugar, de forma introductoria, analizar los cruces, encuentros y desencuentros de los conceptos centrales de la Teoría de sistemas sociopoiéticos de Niklas Luhmann con diversos autores de la filosofía social posestructuralista; segundo, analizar cómo la Teoría de sistemas autopoiéticos analiza y utiliza el concepto de red, central en la ANT en el aspecto de la externalidad de lo humano; y tercero, en función de las limitaciones antropológicas en el sentido comunicativo expuestas por la teoría luhmanniana (periferización de lo humano), y aunque con otra descripción también por la ANT, proponer la utilización de la inclusión antropológica del ser humano con la noción de "autopoiesis reflexiva" en una perspectiva sistémica autorreferencial.

\section{APORTACIONES SISTÉMICAS Y POSESTRUCTURALISTAS: DEFINIENDO SIGNIFICADOS}

Describiremos y analizaremos tres líneas conceptuales procedentes 0 utilizadas en la Teoría de sistemas autorreferenciales y que requieren una explicación más profunda, primero, describiremos lo que entendemos como aportaciones sistémicas autorreferenciales, segundo, expondremos lo que se puede entender como la ambigua etiqueta de filosofía social posestructuralista; y tercero, analizaremos las posibles convergencias y divergencias de la teoría de sistemas luhmanniana con la Actor Network Theory". Dada la amplitud de publicaciones basadas en observaciones empíricas realizadas inspiradas en esta teoría en diversos países, nos limitaremos a las aportaciones realizadas en el ámbito cultural anglosajón y alemán.

R. Inter. Interdisc. INTERthesis, Florianópolis, v.13, n.2, p.39-57 Mai-Ago. 2016 
La ANT, así como otros puntos de vista teóricos (y modelos de aplicación como Decision Support Systems y Actor-System-Dynamics) tienen como elemento común una raíz epistemológica procedente de las matemáticas y la física principalmente, su aplicación en los campos de las ciencias sociales ha sido significativo, siendo la mayoría de aportaciones de antropólogos y sociólogos franceses y británicos (Latour, Callon, Law). Entre estas aportaciones en los últimos años destacan las observaciones e investigaciones empíricas que asumen un referencial teórico-conceptual en una interfaz entre la Teoría de sistemas y la ANT. Nos detendremos aquí en los aspectos teóricos y conceptuales del análisis de redes que pueden establecer un vínculo operativo con la Teoría de sistemas de Luhmann y el posestructuralismo.

\subsection{APORTACIONES SISTÉMICAS AUTORREFERENCIALES}

En el contexto de análisis teórico expuesto, denominamos el proyecto teórico desarrollado por Luhmann como Proyecto Teórico de Sistemas Autorreferenciales, formado por las premisas y conceptos indicados por Lakatos (1980), o sea, Núcleo central, Cinturón protector y Heurística negativa. Carece a nuestro entender, de lo que el pensador y matemático denomina Heurística positiva, es decir, una indicación de las líneas de investigación sobre lo que se puede hacer y unas líneas maestras que indiquen cómo es posible desarrollar el programa, por lo que descartamos la denominación de "Programa de Investigación". Esta situación no es tan solo condicionada por factores externos a la teoría, sino inherente a la misma Teoría de sistemas sociales. Constituye un complejo y abstracto entramado de conceptos y nociones, siendo calificada por algunos sociólogos de "hermética" en sus conexiones de referencia, y de constructivismo radical o incluso solipsista, de tal forma que la estructura de pensamiento y las referencias internas de esta teoría han creado incluso una desorientación. Nuevas aportaciones y la búsqueda de respuestas para las cuestiones planteadas con otros puntos de vista se manifiestan en una situación de continuidad reducida y limitada a reducidos círculos académicos, siendo su situación actual de investigación de debate teórico más bien precaria. Aunque en esta situación posiblemente convergen diversas causas vinculadas a los grupos de poder ideológico y en las líneas de decisión e investigación en la academia.

R. Inter. Interdisc. INTERthesis, Florianópolis, v.13, n.2, p.39-57 Mai-Ago. 2016 
Las controversias actuales de la Teoría de sistema sociopoiéticos y las diversas propuestas orientadas a la idea de superación o distanciamiento teórico del concepto de autopoiesis muestran los intentos por superar su semántica biologista original y su puesta en el entorno de la sociedad de lo humano, para analizar las posibilidades de que el concepto se pueda extender en sus operaciones autorreferenciales en operaciones basadas en comunicaciones sean lingüísticas o contextuales emanadas a través de una conciencia. El seguimiento de la teoría de sistemas sociopoiéticos no es homogéneo, dependiendo en cada país del centro académico y de investigación, y del contexto cultural, al diferir y haberse desarrollado diversas interpretaciones, cruces y convergencias con otras áreas de conocimiento y líneas de pensamiento con abordajes diversos en Alemania, España y América Latina. Una nueva generación de pensadores dialoga y profundiza no sólo el pensamiento de Luhmann, sino también con los avances del debate teórico posestructualista y la teoría sociológica, profundizando los escritos de filósofos como Gilles Deleuze y Michel Callon y de sociólogos como Scott Lash y Bruno Latour principalmente, en el área de la filosofía de la ciencia y con las posibilidades de la performatividad con la sociología económica y cultural (FARIAS; OSSANDÓN, 2006). Este debate teórico se reproduce también en Brasil, en especial con su interpretación y aplicación para la sociología jurídica.

Apuntadas estas especificidades de la teoría de sistemas sociales, con aportaciones sistémicas autorreferenciales nos referimos en este escrito a una serie de trabajos teóricos, que a pesar de sus controversias, discrepancias o "cruces" (FARÍAS; OSSANDÓN, 2009) con algún aspecto de la Teoría de sistemas autopoiéticos de Luhmann, asume como Núcleo central los conceptos de autopoiesis y autorreferencialidad, y en consecuencia de una forma u otra los conceptos de sistema y acoplamiento estructural. Consideramos importante remarcar la dificultad de pode establecer o hablar de una continuidad en la Teoría de sistemas sociales de Luhmann. En el contexto del pensamiento sistémico se diferencia entre la Teoría sociopoiética y la Teoría de sistemas (sistemas abiertos). Ambas lógicas de observación y pensamiento circular han dejado una fuerte herencia que se manifiesta en diversas corrientes, en el ámbito y el trabajo interdisciplinario y continúan un diálogo entre la sociología y otras ciencias, entre la cibernética, las matemáticas, ciencias de la computación y la biología. Un campo

R. Inter. Interdisc. INTERthesis, Florianópolis, v.13, n.2, p.39-57 Mai-Ago. 2016 
especialmente fructífero han sido los estudios y observaciones en el área de la administración, la gestión y la teoría de las organizaciones, mientras que en la Sociología han influenciado decisivamente la Network Theory y la Self-Organzation Theory, esta última en el sentido dado por Leydesdorff (2000) y Fuchs (2002).

\subsection{POSESTRUCTURALISMO}

Con la denominación de posestructuralismo fue definido un movimiento filosófico surgido en los años 1960 y 1970, periodo de desilusión y rechazo de los valores tradiciones de la sociedad burguesa y del surgimiento del feminismo, y que incluye ámbitos tan amplios como la filosofía hasta la historia y la literatura. El término hay que analizarlo con cautela en aspectos teóricos y pragmáticos, ya que crea una serie de dificultades al atribuirle diferentes sentidos, y al estar asociado a la corriente filosófica del posmodernismo. Tampoco pretende etiquetar a filósofos y pensadores pues ellos mismos lo han rechazado (DAVIS; MAQUIS, 2005). Sucede con la filosofía de Derrida, que en sus escritos cuestiona el término deconstrucción y afirma no ser un deconstruccionista, con Lyotard por argumentos similares al intentar definirlo como posmoderno y con Foucault al rechazar el estructuralismo, el posestructuralismo y el posmodernismo, llegando él mismo a afirmar que sus escritos no son estructuralistas.

Mayores dificultades aparecen en la acción de colocar el término posestructuralismo de forma simultánea con posmodernismo, corriente que siempre ha abordado los estudios de organizaciones de forma periférica o incluso peyorativa (FELDMAN, 1997, 1998). Su influencia es controvertida ya que el posestructuralismo es visto a menudo como un "posicionamiento disidente", como por ejemplo respecto a las ciencias en general y a los valores establecidos (JAMES, 2005). Aunque algunos autores delimitan este término a una aparente superación o radicalización del estructuralismo - la línea divisoria con el posestructuralismo es un intento de establecer una línea temporal - sigue siendo arbitrario y por lo tanto sujeto a lecturas semánticas (TADAJEWSKI; MACLARAN; PARSONS, 2011; WILLIAMS, 2005). Como posible definición o intento de otorgar un sentido al posestructuralismo, es posible vincularlo a los postulados sociológicos del interaccionismo simbólico (Berger y Luckmann) así como al predominio de la observación de segundo orden. 
Entre estas se destacan los intentos de restablecer una praxis con un diálogo filosófico de esta corriente con el feminismo (DONE; KNOWLER, 2011, p.841-852), y el management de organizaciones públicas, destacando la gestión crítica (ALVESSON; DEETZ, 1999), y la incorporación de las nociones de gestión estratégica.

\subsection{LA "ACTOR NETWORK THEORY"}

La "Actor Network Theory" (ANT) es un punto de vista constructivista, cuyos orígenes se remontan a la investigación técnica y científica, y que tiene por objetivo proponer un nuevo paradigma transdisciplinar, y según Crawford (2004): "for exploring collective sociotechnical processes" (CRAWFORD, 2004), en los que se ha prestado una atención especial a los estudios de la sociología de la ciencia y la tecnología. Tres principios centrales configuran la ANT: primero, la no formulación de ninguna hipótesis causal a priori sobre la naturaleza de las redes, o condiciones causales sobre la exactitud de las acciones de los actantes; segundo, el principio orientador de la red es la simetría, el empleo de un solo cuadro explicativo al interpretar actantes, humanos y no humanos; tercero, la libre asociación, o sea, el abandono de cualquier distinción entre fenómeno natural y social. En base a estos principios el papel del observador debe reducirse a la observación de la red en una limitación espacial, o sea, sin necesidad de desplazamiento para examinar los "actantes" y las organizaciones o integrantes no humanos.

La ANT describe una materialidad relacional que al tratarse también de la extensión de la semiótica, (o sea, observación del signo y la producción del sentido) parte del presupuesto de que todas las entidades que forman parte de la red ("actantes") alcanzaron significación en relación con los demás. Agrupa un grupo de filósofos, sociólogos y consultores y representa una respuesta crítica a los efectos reificadores del construccionismo social a la hora de explicar lo social. Se inserta en el contexto cambio paradigmático ya iniciado en la década de 1980, con la popularización de conceptos nuevos en las ciencias sociales como "autopoiesis", "autorreferencialidad", “complejidad", “catástrofes”, “red”, “autoorganización”, "rizoma" o "semiótica social". 
Aunque con la emergencia de estos conceptos se anunciaba tan solo el comienzo de lo que algunos autores denominaron la "demolición minuciosa y pertinaz" de las dicotomías que tradicionalmente marcaron la observación y el análisis de la sociedad. Su emergencia en las ciencias sociales causó reacciones de estupor entre muchos de los sociólogos que analizan la sociedad desde perspectivas y paradigmas muy arraigados en las ciencias sociales (DOMÉNECH; TIRADO, 1998, p.12). Estos conceptos son un intento de establecer una interconexión lógica que se alejase de los imperativos funcionalistas y neo-marxistas entre ciencia, tecnología y sociedad, y de superar la clásica oposición entre agencia / estructura, (y otras como: naturaleza / sociedad, macro / micro, y objeto / humano) dándose un nuevo impulso a la teoría sociológica y con implicaciones directas en las teorías organizacionales.

Entretanto, ya entrada la segunda década del siglo XXI, los conceptos han cambiado para la noción de "social cyborgs" (cybernetic organisms) que engloban a una serie de nuevas ideas, nociones y conceptos para pensar lo social tales como: "ciberespacio", "ciberdemocracia", "contornos virtuales", "redes ciudadanas", "cibercultura”, "ciberpsicología”, “Cyborgian Territorialisations", "copresencia”, "nonlinear", por citar algunos. En este contexto de cambio epistemológico radical se encuentran algunas de las ideas centrales que componen la "Actor-Network-Theory" y la "Sociología sinétrica", así como también la crítica dirigida a la ciencia y la tecnología como sustento del patriarcado realizada por parte del feminismo radical. Fundamentada en el Principio se Simetría, Bloor (1976) consideró elevarla a un status epistemológico de Programa Fuerte siendo calificado de "the manifesto of Schottisch Scientism" (BOON, 1979) sobre la teoría del conocimiento desarrollada en la Escuela de Edimburgo. Sociólogos, filósofos y pensadores cuya obra se enmarca en el posestructuralismo utilizaron nuevas denominaciones de la ANT, incluso con definiciones tan rebuscadas como "Ontología del Actante-Rizoma".

En esta nueva línea argumentativa de pensamiento, el filósofo francés Latour (2005) se refiere a la emergencia de una "Sociología de las asociaciones" (sociology of association), Callon (1986) se refiere a "Sociología de la traducción" (sociology of translation). El filósofo mexicano DeLanda (2006) complementa y amplía estas descripciones sociales, y afirma que la presentación de la sociedad se basa en un "infinito ensamblarse y desensamblarse de actuantes humanos y no humanos" en un

R. Inter. Interdisc. INTERthesis, Florianópolis, v.13, n.2, p.39-57 Mai-Ago. 2016 
marco referencial teórico de "Teoría de los ensamblajes y la complejidad social", en la que se manifiesta una profunda brecha respecto a las teorías sociológicas clásicas y contemporáneas.

\section{SEMÁNTICAS DE LOS TÉRMINOS DE "ACTOR" Y "RED” EN LA ACTOR- NETWORK-THEORY}

Los estudiosos de la ANT han formulado una serie de presupuestos teóricos, en los que los términos "actor" y "red" constituyen un intento por sobrepasar la distinción habitual sociológica entre acción y estructura (o entre agencia y estructura). Con la idea de red se incluye una serie de actividades integradas que los "actantes" realizan, los cuales con cada enlace o nodo (en el caso de la informática y la telecomunicación) se deriva semióticamente en aspectos observables de variables y contingencia. En un plano analítico el objeto principal de atención de la ANT es observar la morfología, estructura, organización de la red.

La perspectiva posestructuralista del enfoque de ANT proviene principalmente del filósofo francés Bruno Latour. Para este filósofo una red consiste en: "seguir las cosas a través de las redes en que ellas se transportan, describirlas en sus enredos" (LATOUR, 2004, p.379). Sus críticas y distanciamiento de la teoría de sistemas provienen de cuatro "pequeños problemas". El primero, comienza con la propia definición de ANT, puesto que los conceptos de actor y red se contraponen a la misma ANT al tratarse de una propuesta básicamente técnico-metodológica. Segundo, la misma autodefinición de ANT como una "teoría", puesto que se trata ante todo de un método. Tercero, el concepto de "actor" utilizado se diferencia del "actor social". En la ANT se refiere a sujetos humanos como "entidades animadas" (referidos por los efectos de sus acciones) y a actores no humanos referidos como "entidades inanimadas", puesto que lo que no deja huella no se considera actor. Cuarto, el concepto mismo de "red" lo desvincula de la cibernética, asumiéndole una semántica de flujos, movimientos, alianzas y los cambios provocados. Para Latour y Callon (1986), no existe de antemano un mundo de las "cosas" y un mundo de los hombres ya que la naturaleza y la sociedad son efectos de redes heterogéneas. Lo "social", se trata de una red heterogénea formada no solo por personas humanas, sino también por no humanos, siendo las comunicaciones y los objetos el medio de

R. Inter. Interdisc. INTERthesis, Florianópolis, v.13, n.2, p.39-57 Mai-Ago. 2016 
la comunicación. La premisa de que la sociedad está compuesta por comunicaciones evidencia las coincidencias con los postulados sistémicos autorreferenciales.

A pesar de Latour ser uno de los principales impulsores de la ATN, posteriores aportaciones han creado una serie de controversias, al mostrarse sumamente crítico, llegando a afirmar: "... hay cuatro cosas que no funcionan con la teoría del actor-red; la palabra actor, la red de la palabra, la palabra teoría y el guión! Cuatro clavos en el ataúd" (LATOUR, 1999, p.15). Posteriormente en una "introducción" asume que con la ANT se trata de una definición "torpe, sin sentido y que no merece ser conservado", derivado de la utilización de término que crean confusión en la sociología y a los propios actores a los que se pretende estar observando. Las clásicas dicotomías sociológicas (macro / micro y objeto / humano actor) aparecen como extremamente problemáticas e incluso innecesarias para los teóricos de la ANT. Latour considera necesario colocar en un "nivel ontológico plano" estas distinciones evitando de esta forma que un actor "macro" sea visto como "agencia", mientras que el "micro" sea visto como un objeto.

Latour considera que se han desarrollado tres recursos o posibilidades para dar una respuesta a la presentación social de las agencias (LATOUR, 1996). El primero, es atribuirles aspectos y elementos biológicos y vincularlas con la biología y la naturaleza. El segundo, es concederles un status social -la sociabilidad- e intentar vincularlas al tejido social. El tercero, es considerarlas como una construcción semiótica y relacionar la agencia con la construcción del significado. Los estudios de la ciencia se han visto imposibilitados de diferenciar claramente los tres recursos, puesto que seres biológicos minúsculos son al mismo tiempo naturaleza, social y discurso ya que se trata de entidades reales, vivas y semióticas. La teoría del ART de Callon es un intento de crear una serie de nociones y conceptos específicos para intentar ofrecer respuestas a esta nueva situación.

En diversas publicaciones y estudios se ponen de manifiesto una serie de críticas y malentendidos de la ANT. La primera se refiere al concepto de red. Con una red, que puede tratarse de una red técnica o informática, se trata tan sólo un posible estado final en la que participan "actantes", puesto que un "actor-red" puede tratarse de un ser humano y no de un "actante" técnico por lo que aparecen aspectos relativos a la naturaleza humana (como la imprevisibilidad o la toma de

R. Inter. Interdisc. INTERthesis, Florianópolis, v.13, n.2, p.39-57 Mai-Ago. 2016 
decisiones no estratégicas), como ya ha constatado Tom Hughes en su estudio histórico Networks of Power (1983) (Redes de poder). Se trata de actores-redes en los que a partir de la historia sólo algunos de sus elementos estabilizados terminan transformándose en redes en un sentido técnico. La definición de las redes sigue siendo una proyección parcial de un "actor-red".

La segunda está relacionada con la propia concepción dada a la Network Actor Theory con lo que se desvincula del estudio propio de las redes sociales. En los estudios que focalizan las "redes sociales" se desvinculan de las preocupaciones inherentes con las relaciones sociales de los actores humanos individuales (frecuencia, distribución, homogeneidad, la proximidad). La ANT surgió como un rechazo a otras teorías que focalizaban macro conceptos en un nivel global (instituciones, organizaciones, e incluso estados) con el objetivo de atribuirles un significado más próximo a los fenómenos sociales focalizando las asociaciones.

\section{TEORÍA SISTÉMICA DE LUHMANN Y LA PROCURA DE RESPUESTAS EN LA ACTOR-NETWORK-THEORY}

En los últimos años son cada vez más numerosas las publicaciones que establecen un vínculo teórico y operacional entre la teoría de Luhmann y la "Actor Network Theory" (WHITE, 2007; 2008), en especial en el ámbito de la lengua alemana (HOLZER, 2011; REISER-KAPELLER, 2011; HOLZER; SCHMIDT, 2009; KNEER; NASSEHI, 2000). Las diversas propuestas dialógicas entre los estudiosos de la Teoría de sistemas luhmanniana y la ANT han tenido como fuente común observaciones empíricas focalizadas en temas específicos en las que se observan los procesos complejos de auto-organización y heterogenización de diversos fenómenos, entre los que se destacan para la comprensión del mundo rural (NOE; ALRøE, 2006), la comprensión teórica de la obra de arte y su conexión interna y externa a través de un dialogo con Bourdieu, Luhmann, Hennion y Latour (ALBERTSEN; DIKEN, 2003), o las posibilidades de interconexión con el paradigma relacional (SCHMITT, 2008).

Luhmann ofrece respuestas teóricas y conceptuales a la situación de ambigüedad de la sociología clásica, no como habitualmente había sido, o sea, a partir de la descripción y definición de un centro en el que el hombre y su acción son

R. Inter. Interdisc. INTERthesis, Florianópolis, v.13, n.2, p.39-57 Mai-Ago. 2016 
el punto de referencia, sino desde su entorno. Este hecho creó desconfianza con las tradiciones humanista, dialéctica o fenomenológica de la sociológica tradicional. Como consecuencia, el proyecto teórico luhmanniano difícilmente dispuso de posibilidades conceptuales y analíticas de diálogo con otras teorías que surgieron en el mismo espacio temporal de la década de los años 80 y 90, como la Teoría de la estructuración de Giddens, o con la propuesta de presentación de la sociedad en base a la dicotomía dependencia / autonomía, de Castoriadis (1975), autopresentándose como autoexcluyentes con la teoría sistémica. Esta falta de diálogo y debate colectivo durante estas décadas no es solamente un problema de la complejidad y falta de tradición y rompimiento de la Teoría de sistemas autorreferenciales con la teoría sociológica clásica, sino que el mismo contexto de debate teórico no fue el que más posibilidades brindó. Los sociólogos no iniciaron diálogos suficientemente productivos dirigidos a un esfuerzo colectivo más amplio para analizar otras posibilidades de descripción de la sociedad y procurar otras semánticas a los intocables conceptos luhmannianos calificados de "potencia teórica" (FARÍAS; OSSANDÓN, 2009, p. 5).

Emergen ciertas coincidencias en la semántica de algunos conceptos entre los puntos de vista teóricos entre Luhmann y White, aunque la premisa inicial entre ambos pensadores procede de premisas iniciales radicalmente opuestas. Mientras que para Luhmann las personas son "construcciones comunicacionales" que solo aparecen en el proceso de comunicación en el entorno del sistema, para White, en un intento para superar los dualismos tradicionales de la sociología, su supuesto básico es el denominado "imperativo autocategórico", el cual rechaza los intentos de explicar la conducta humana en base a los atributos de los actores (individuales 0 colectivos), aunque no excluye sus propias identidades. En su concepto de "relational sociology", atribuye a las redes unas propiedades fenomenológicas, o una perspectiva relacional, al ser creadas y formadas por personas con capacidad de darles significado: "network are phenomenological realities as well as measurement constructs"3.

A pesar de asumir una serie de premisas iniciales comunes con la teoría luhmanniana, sigue el problema ambiguo de la división entre humano y no humano que realizan los teóricos la ANT: "no existe de antemano un mundo de las cosas y

${ }^{3}$ Harrison White, Identity and Control. Publicado originalmente en 1992, revisado y ampliado en 2007.

R. Inter. Interdisc. INTERthesis, Florianópolis, v.13, n.2, p.39-57 Mai-Ago. 2016 
un mundo de los hombres" (CALLON, 1986), o de la periferización del "ser humano" en la teoría sociopoiética de Luhmann. Para Latour "lo social" trata de una red heterogénea formada no solo por personas, mientras que para Luhmann se trata de comunicaciones sistémicas no realizadas por "seres humanos corporeizados" (LUHMANN, 1984, p.236). Aceptamos el presupuesto inicial según el cual la sociedad está formada por comunicaciones, siendo estas comunicaciones emitidas tanto por sujetos como por sistemas, y mantenemos la idea central de que los sistemas están regidos en sus operaciones inicialmente por "sistemas psíquicos" y en un nivel superior por "sistemas de conciencia", definidos de esta forma por Luhmann aunque con diferentes atribuciones autorreferenciales.

La idea de una argumentación comunicativa destaca el intento de atribuir a la idea de autopoiesis luhmanniana una lógica de autoindexicalidad - "autopoiesis indexical"-, y pretende explorar la posibilidad de inclusión del concepto de "indexicalidad" proveniente de la etnometodología y los estudios conversacionales de Harold Garfinkel. La lógica de la operación se realiza mediante la identificación del cierre de sus operaciones y los procesos relacionales en la contextualidad de las operaciones que tienen lugar en la interacción, y considera la existencia de una autopoiesis sui generis como propiedad de los sistemas de interacción como "entramados complejos" (ROBLES, 2004). Aunque no directamente vinculado con la idea autorreferencial de Luhmann, las ideas y nociones de racionalidad y comunicación sistémica han contribuido y sido aplicados además de la ANT, en la Teoría de los juegos, la Teoría de la decisión y la "Self-organization theory" (LEYDERSDORFF, 2000).

\section{DERIVACIONES TÉCNICAS DE LA ANT Y LA TEORÍA DE SISTEMAS}

En la segunda década del siglo XX la Teoría de sistemas sociales de Luhmann y la ANT han sido fructíferas especialmente en el campo de las teorías organizacionales, de las cuales han derivado diversas posibilidades observacionales y de análisis de redes utilizadas y adaptadas también como propuestas de consultorías de organizaciones. Entre estas se destacan la Decision Support Systems y Actor-System-Dynamics, teniendo ambas como base común la teoría de sistemas.

R. Inter. Interdisc. INTERthesis, Florianópolis, v.13, n.2, p.39-57 Mai-Ago. 2016 
- Entre los nuevos enfoques del análisis de redes se destaca el denominado Decision Support Systems (DSS). Inspirado en ideas procedentes de la cibernética y la teoría de sistemas, se trata de un sistema de información basado en cálculos realizados por computadoras o por personas, y que tiene como objetivo apoyar la toma de decisiones organizativas. La utilización de la DSS no solo se ha enmarcado como un proceso social de adopción o proceso técnico de tomas de decisiones organizacional (básicamente en organizaciones económicas), sino que también se ha aplicado e institucionalizado en varias organizaciones de manejo forestal en el estado alemán de Renania. Investigaciones innovadoras utilizan el modelo teórico de la Actor Network Theory con el diseño de un DSS, con el objetivo de comprender el funcionamiento de las redes de trabajo gracias al enfoque interpretativo (BOERBOOM; FERRETTI, 2014). La DSS puede ser exclusivamente informatizada, con intervención humana o una combinación de ambos y sirve para la toma de decisiones en la gestión organizacional y para operaciones y niveles de planificación, ya que se trata de un entorno rápidamente cambiante y no fácilmente específico de antemano.

- Por otro lado, el enfoque Actor-System-Dynamics (ADS), está inspirado en la idea funcionalista de grupo de Parsons y sobre la base de niveles sociales grupales y el enfoque de redes, la propuesta teórica ADS ha sido calificada como un "Research program", aplicándose y desarrollado en los análisis de las instituciones, organizaciones y sociales. Se trata de un enfoque cuyas raíces provienen de la teoría de los sistemas sociales y cuyo sistema lo constituye el "grupo", y un contexto ecológico y social formado por "resource base, rule regime base, agential base e interactions, productions, outputs e outcomes" (BURNS; CORTE; MACHADO, 2014). Aunque estos puntos de vista teóricos son aportaciones interesantes para la observación de grupos sociales, al disponer todavía de pocos resultados de observaciones empíricas, su efectividad en las observaciones debe ser objeto de futuros análisis.

\section{CONSIDERACIONES FINALES}

White ya ha explicado en diversos trabajos que uno de los principales giros en la ANT actual ha sido intentar comprender cómo se constituyen mutuamente,

R. Inter. Interdisc. INTERthesis, Florianópolis, v.13, n.2, p.39-57 Mai-Ago. 2016 
conexiones sociales y los conjuntos de historias (WHITE, 1995; WHITE; MOHR, 2008; WHITE; GODART, 2008). Entretanto, también algunos estudiosos de la ANT ya apuntan hacia los "límites" de la ANT (HÄUSSLIG, 2009). Sin embargo, el problema de asumir los conceptos luhmannianos de autopoiesis todavía no está satisfactoriamente resuelto puesto que la semántica del estudio y significado de las redes mantiene fundamentos fenomenológicos en sus comunicaciones. Diversos trabajos apuntan en esta dirección, como la propuesta de Stephan Fuchs de "antiessentialism" con dinámicas micro-sociológicas, cuyas premisas básicas son "personas", "humanismo", "naturaleza" y "sociedad".

Somos con ello conscientes de que la descripción crítica comparada entre la Teoría de Sistemas Sociales y la ANT abre posibles potenciales de conexión e interrelación que sobrepasan las descripciones expuestas en este artículo. Son cada vez más numerosas las observaciones que vinculan sistemas, redes e identidades como nuevo fundamento para explicar lo social. Un ejemplo pueden ser los diversos grupos sociales urbanos y sus identidades en el contexto de redes y la procura de identidades propias. Estas se apoyan en la ANT y sus fuentes fenomenológicas (White) y en la Teoría de sistemas de Luhmann. Las redes están constituidas de Dyaden que emergen entre los nudos de la red, y que a su vez establecen relaciones entre las personas. El asumir los conceptos y nociones de la Teoría de sistemas autorreferenciales puede conducir a una sofisticación de los estudios y observaciones:

- Sugerir que las Dyaden puedan ser pensadas y operacionalizadas de forma autopoiética.

- Sugerir que los sistemas puedan ser tratados de nudos, los cuales en la red disponen de una identidad.

- Estos nudos pueden disponer de una identidad y a partir de operaciones internas son autoobservables.

- Los nudos pueden operar a su vez de forma de cierre autopoiético, de delimitación con el entorno o de abertura con el entorno.

Estos cambios no son casuales, sino que de una forma $u$ otra sintetizan y intentan dar un sentido semántico, y a su vez a las ambigüedades y controversias en la redefinición al atribuir a un actor genérico -"actante"- un tipo de acción, y la posibilidad dual de presentarlo como un ser humano y no humano. Estos cambios

R. Inter. Interdisc. INTERthesis, Florianópolis, v.13, n.2, p.39-57 Mai-Ago. 2016 
paradigmáticos y de teorización se han aplicado en observaciones que ponen de manifiesto la heterogeneidad constitutiva en prácticas identitarias que no pueden ser subsumidas en una única dirección o plano como es el caso de los movimientos sociales.

Surgen importantes cuestiones epistemológicas sobre la propuesta de Latour (2008), quien declara que la sociología no se puede limitar en la ciencia de lo social, sino que "debería hacerse cargo" de describir las asociaciones entre elementos heterogéneos como son seres humanos, animales, objetos, materiales. Su propuesta de "Sociología de las asociaciones" no se ha llevado a cabo ya que la dimensión social de un fenómeno que objetivamente puede no existir a priori no se puede utilizar como punto de partida para investigación.

R. Inter. Interdisc. INTERthesis, Florianópolis, v.13, n.2, p.39-57 Mai-Ago. 2016 


\title{
TEORIA DE SISTEMAS AUTOPOIÉTICOS E A "ACTOR NETWORK THEORY": PARA UMA CONVERGÊNCIA PÓS-ESTRUTURALISTA?
}

\section{Resumo:}

Durante anos houve um interesse crescente entre os sociólogos para estabelecer uma relação entre a teoria dos sistemas sociais autorreferenciais de Nikolas Luhmann com os referenciais teóricos que compõem os conceitos da filosofia social pós-estruturalista. Embora haja uma autodefinição estrutural-funcional comum, observam-se diferenças inevitáveis entre a teoria dos sistemas de Luhmann e o construtivismo Actor Network Theory.

Palabras-chave: Teoría de sistemas autopoiéticos. Actor Network Theory. Comunicações. Ator

\section{AUTOPOIETIC SYSTEMS THEORY AND THE "ACTOR NETWORK THEORY": TOWARDS A POST-STRUCTURALIST CONVERGENCE?}

\begin{abstract}
:
In recent years a growing interest among sociologists in establishing a link between the theory of self-referential social systems by Niklas Luhmann to the theoretical frameworks that make up the social poststructuralist philosophy manifests. As autoobservables systems and differentiation of human actors and nonhuman, unavoidable differences appear between Luhmann's systems theory and constructivism of ANT
\end{abstract}

Keywords: Autopoietic Systems Theory. Actor Network Theory. Communications. Actor. 


\section{REFERENCIAS}

ALBERTSEN, Niels; DIKEN, Bülent. Artworks' Networks - Field, System or Mediators? Department of Sociology, Lancaster University, Lancaster Disponível em: <http://www.comp.lancs.ac.uk/sociology/papers/Albertsen-Diken-ArtworksNetworks.pdf> Acesso em: 22 fev. 2010

BLOOR, David. Knowledge and Social Imagery. Henley: Routledge \& Kegan, 1976.

BOON, Louis. Knowledge and Social Imagery by David Bloor, The British Journal for the Philosophy of Science, v. 30, n.2, p. 195-199, jun. 1979.

BÜHL, Walter. Grenzen der autopoiesis. Vordenker, Oktober. Disponível em: <http://www.vordenker.de/buehl/wlb grenzen-autopoiesis.pdf > Acesso em: 3 ago 2013

CALLON, Michel. Some Elements of a Sociology of Translation: Domestication of the Scallops and the Fischermen of St. Brien Bay. In: John LAW (Org.) Power, Action a Belief: A New Sociology of Knowledge? London: Routledge \& Kegan Paul, 1986, p. 196-223.

CALLON, Michel; LATOUR, Bruno. Unscrewing the big Leviathan: how actors macrostructurereality and how sociologists help them to do so. In: K. KNORR-CETINA; A. V. CICOUREL (Org.) Advances in social theory and methodology: Toward an integration of micro- and macro-sociologies. Boston, London and Henley: Routledge \& Kegan, 1981, p. 277-303.

CASTORIADIS, Cornelius. L'Institution imaginaire de la société. Paris: $\underline{\text { Seuil, }}$ 1975.

COOPER, Paul, S. Regulating Hybrid Monsters? The Limits of Latour and Actor Network Theory. ARENA, 2008, p. 305-330.

CRAWFORD, Cassandra. Actor network theory. Ritzer, Georg. Encyclopedia of social theory. Thousand Oaks: Sage Publications Inc., 2004.

DE OLIVEIRA, Adriano Messias. Caminhos e Descaminhos da Intersubjetividade. Os laços sociais e a construção da identidade. [201?] Disponível em:

R. Inter. Interdisc. INTERthesis, Florianópolis, v.13, n.2, p.39-57 Mai-Ago. 2016 
http://bocc.ubi.pt/pag/oliveira-adriano-intersubjectividade.pdf Acesso em: 17 nov. 2013

DELANDA, Manuel. A New Philosophy of Society: Assemblage Theory and Social Complexity. London/New York: Bloomsbury Academic, 2006.

DELEUZE, Gilles. ¿En qué se reconoce el estructuralismo? In: Deleuze, La isla desierta y otros textos. Textos y entrevistas (1953-1974). Valencia: Pre-Textos, 2005.

DOMĖNECH, Miquel; TIRADO, Francisco Javier. Sociología sinétrica. Ensayos sobre ciencia, tecnología y sociedad. Barcelona: Gedisa, 1998.

EMIBAYER, Mustafa. Manifesto for a Relational Sociology, American Journal of Sociology, v. 103, n.2, p.281-317, 1997.

FARIAS, Ignacio; OSSANDÓN, José (edit.). Observando Sistemas. Nuevas apropiaciones y usos de la teoría de Niklas Luhmann. Santiago de Chile: RilEditores-Fundación Soles, 2009.

HABERMAS, Jürgen. El discurso filosófico de la modernidad. Madrid: Taurus, 1993.

HARAGAW, Donna. A Cyborg Manifesto Science, Technology, and SocialistFeminism in the Late Twentieth Century. In Simians, Cyborgs and Women: The Reinvention of Nature. New York: Routledge, 1991, p.149-181.

HARMAN, Graham. Prince of Networks: Bruno Latour and Metaphysics. Unpublished Manuscript, 2007.

LATOUR, Bruno. Reassembling the Social: An Introduction to Actor-NetworkTheory. Oxford: Oxford University Press, 2005.

LATOUR, Bruno. On actor-network theory. A few clarifications plus more than a few complications, Soziale Welt, v.47, 1996, p.369-381.

LATOUR, Bruno. Science in Action: How to Follow Scientists a Engineers Through Society. Milton Keynes: Open University Press, 1987. 
LATOUR, Bruno. On Recalling ANT, The Sociological Review, v. 47, n.51, p. 15-25, 1999.

LEYDESDORFF, Loet. "Structure"/"Action" Contingencies and the Model of Parallel Distributed Processing, Journal for the Theory of Social Behaviour, n. 23, p. 47$77,2000$.

LUHMANN, Niklas. Organisation und Entscheidung. In: LUHMANN, N.

Soziologische Aufkrärung. Opladen: Westdeutscher Verlag, 1981.

LUHMANN, Niklas. Soziale Systeme. Grundriß einer allgemeinen Theorie.

Frankfurt a.Main: Suhrkamp Verlag, 1984.

McLEAN, Chris; HASSARD, John. Symmetrial absence/symmetrical absurdity: critical notes on the production of actor-network accounts, Journal of Management Studies, v. 41, n.3, p.493-519, 2004.

NOE, Egon; ALRØE, Hugo Fjelsted. Combining Luhmann and Actor-Network Theory to see Farm Enterprises as Self-organizing Systems. Danish Research Centre for Organic Farming (DARCOF), Paper to be presented at: The Opening of Systems Theory" in Copenhagen May 23-25 2003.

SCHMITT, Marco. Systems and Networks. Theoretical Combinations Inside the Relational Paradigm, 4th UK Social Networks Conference, 2008. Disponível em: $<$ https://sites.google.com/site/businessnetworkresearch/Home/papers-2/Systemsand-Networks-Theoretical-Combinations-Inside-the-Relational-Paradigm> Acesso em: 25 jul. 2013

WHITE, Harrison C. Identity and Control. A Structural Theory of Social Action. Princeton (NJ.): Princeton University Press, 1992.

WHITE, Harrison C.; Frederic GODART. Linking Networks and Domains: Cultural and Discursive Formations in Context, Manuscript for Manchester talk, Jan. 2008.

\section{Artigo}

Recebido em 31 de Agosto de 2015

Aceito em 11 de Abril de 2016

R. Inter. Interdisc. INTERthesis, Florianópolis, v.13, n.2, p.39-57 Mai-Ago. 2016 